\title{
Internal radiation dose and modeling codes in nuclear medicine: a fresh look at old problems
}

\begin{abstract}
In the Medical Internal Radiation Dosimetry (MIRD) method, the doses absorbed in the target organs are estimated as a function of activities accumulated in a source organ. Internal dosimetry codes help standardize dose calculations using recognized models, phantoms, and dosimetry techniques. In nuclear medicine community, the aim is to perform calculations to obtain dose estimates for various organs of the body once the kinetics of a radiopharmaceutical agent is established. Accurate dosimetry for each investigation is needed to optimize the use of various alternative nuclear medicine techniques and to estimate the radiation exposure and risk from each procedure. This paper reviews some basic concepts of internal dosimetry codes, key features and difficulties of existing classes for internal radiation dosimetry and modeling codes in nuclear medicine, and the phantoms associated with them.
\end{abstract}

Keywords: mird method, internal dosimetry, phantoms, nuclear medicine

\author{
Volume 4 Issue 5 - 2017 \\ Susie Medeiros Oliveira Ramos,' Sylvia \\ Thomas, ${ }^{2}$ Monica Araujo Pinheiro, ${ }^{2}$ Francisco \\ de Assis Romeiro Figueiroa Benicio Coelho, ${ }^{2}$ \\ Marta de Souza Albernaz, ${ }^{2}$ Clara Lorena \\ Gloria dos Santos, ${ }^{2}$ Mirta Bárbara Torres \\ Berdeguez, ${ }^{3}$ Fernando Eduardo Zikan, ${ }^{4}$ \\ Priscylla da Costa Medeiros,' Debora da \\ Cunha Pereira Sampaio, ${ }^{5,6}$ Bruce Cesar de \\ Toledo, ${ }^{6}$ Lidia Vasconcellos de Sá, ${ }^{6}$ Sergio \\ Augusto Lopes de Souza' \\ 'Department of Radiology, University of Clementino Fraga Filho \\ Hospital, Brazil \\ ${ }^{2}$ Department of Nuclear Medicine, University of Clementino \\ Fraga Filho Hospital, Brazil \\ ${ }^{3}$ Department of Nuclear Engineering, University of Federal Rio \\ de Janeiro (UFRJ), Brazil \\ ${ }^{4}$ Department of Physiotherapym, University of Clementino \\ Fraga Filho Hospital, Brazil \\ 5University of Estácio de Sá, Rio de Janeiro, Brazil \\ ${ }^{6}$ Department of Medical Physics, Brazilian Nuclear Energy \\ Commission, Brazil
}

Correspondence: Susie Medeiros Oliveira Ramos, Federal University of Rio de Janeiro, Clementino Fraga Filho University Hospital, Radiology Department, Rua Professor Rodolpho Rocco, 255, llha do Fundão - Zip Code: 2194 I-913, Rio de Janeiro - RJ - Brazil,Tel 5521 39386274, Fax 552139386274 , Email medeiros.susie@gmail.com, susie@ird.gov.br

Received: November 0I, 2017 | Published: November 22, 2017
Abbreviations: MIRD, medical internal radiation dosimetry; DT, absorbed dose to a target region; CTL, computed tomography; SPECT/CT, single photon emmission tomography/computed tomography; PET/CT, positron emmission tomography/computed tomography; 3D MRI, tridimensional magnetic resonance; NURBS, non-uniform rational b-spline surface; SI, international System; MC, monte carlo simulation; FDA, food and drug administration; ICRP, international commission on radiological proteCtion; TLD, thermoluminescent dosimetry technique

\section{Introduction}

Absorbed doses of different organs can be estimated by different methods such as Medical Internal Radiation Dosimetry (MIRD). In this method, the doses absorbed in the target organs are estimated as a function of activities accumulated in a source organ and it provides a corrected mathematical estimated dose. ${ }^{1,2}$ Internal dosimetry codes were designed primarily to lighten manual dose calculations, frequently using tables from MIRD Radionuclide Data and Decay Schemes, ${ }^{3}$ and ICRP Publication $38 .{ }^{4}$

With these codes, basic dose calculations could be made in seconds instead of hours. Another purpose of these codes was to help standardize dose calculations using recognized models, phantoms, and dosimetry techniques. In nuclear medicine community, the aim is to perform calculations to obtain dose estimates for various organs of the body once the kinetics of a radiopharmaceutical agent is established. The administration of radiopharmaceuticals to humans for diagnostic, therapy or research purposes is a well-established and developing branch of the nuclear medicine practice. New methods and radiotracers are continually introduced. Accurate dosimetry for each investigation is needed to optimize the use of various alternative nuclear medicine techniques and to estimate the radiation exposure and risk from each procedure. ${ }^{5}$

Many research groups that work in internal dosimetry have been developing their own tools, and day by day new software is being published. ${ }^{6}$ This paper reviews some basic concepts of internal dosimetry codes, key features and difficulties of existing classes for internal radiation dosimetry and modeling codes in nuclear medicine, and the phantoms associated with them.

\section{Discussion}

\section{Basic Concepts}

To understand the calculations performed to obtain the total absorbed dose (DT) to a target region through a certain code, firstly 
the user needs to know basic internal dosimetry concepts, such as target and source regions, $\mathrm{S}$ values, and cumulated activity in each organ. Herewith the main definitions are as follows:

\section{Source region}

Region that holds a radiopharmaceutical for a specific amount of time related to the biological half-life of the pharmaceutical, and it is emitting ionizing radiation to other regions nearby. ${ }^{7}$

\section{Target region}

Region that is exposed to the ionizing radiation from the source regions.

\section{Cumulated activity}

The level of activity obtained at different times after injection, plotted against time, gives a time-activity curve for a particular organ. The integral of this curve gives the total number of disintegrations or the cumulated activity for the region, which must be determined for each patient. $^{7}$

\section{S value}

The absorbed dose to a target region per unit cumulated activity in the source regions.

\section{Absorbed dose}

Expressed in units of Gray ("rad" in older texts; $1 \mathrm{~Gy}=100 \mathrm{rad}$ ), it is defined as the energy absorbed in a particular mass of tissue, divided by the tissue mass. In internal dosimetry, DT is the goal of the calculations, and it is defined as the sum of mean dose contributions to the target from different source regions. ${ }^{7}$

\section{Stylized, voxel, hybrid and physical phantoms - why do they exist?}

It is well known that a precise representation of the human body is important for accurate internal dosimetry. The ideal situation would be to use the diagnostic image of the patient as anatomic model to estimate doses received by the diagnostic image itself or by a previous nuclear medicine treatment. Such "patient-specific" anatomic models can and are used in a research-level, where the Computed Tomography (CT) portion of Single Photon Emmission Tomography/Computed Tomography) SPECT/CT or Positron Emmission Tomography/ Computed Tomography (PET/CT), or even Tridimensional Magnetic Resonance (3D MRI) as shown by Berdeguez et al. ${ }^{8}$ could be used for radiation transport simulation and new dose planning methodologies. ${ }^{8}$ Problems of image-based patient-specific models include the lack of whole-body coverage in most cases and the need to segment those images to quantify organ absorbed doses separately.

Anthropomorphic phantoms exist to simulate the human body's reaction to ionizing radiation, and allow whole-body or partial dosimetry in the organ of interest. The stylized phantoms have the size and form of the body and its organs are described by mathematical expressions (combinations/intersections of planes, circular and elliptical cylinders, spheres, cones, etc.). ${ }^{9}$ Voxel phantoms are based on digital images recorded from CT or MRI. ${ }^{10}$ Hybrid phantoms are the combination of both, stylized and voxel phantoms. The hybrid approach to a computational phantom construction is based on nonuniform rational B-spline (NURBS) surface animation technology that has the advantages of both former phantom generations. ${ }^{11}$ These three generations are shown in Figure 1.

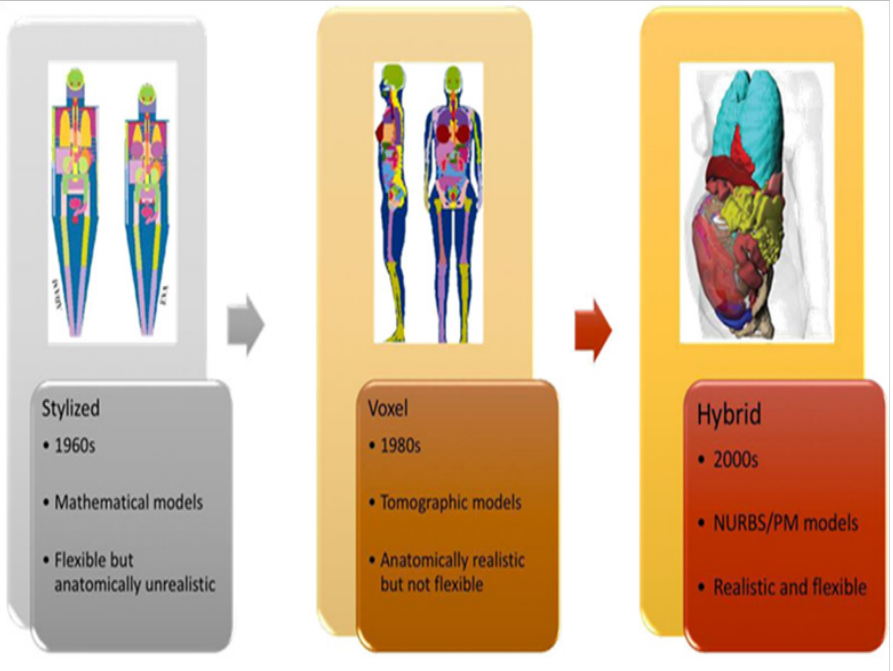

Figure I From left to right, examples and characteristics of the first, ${ }^{13}$ the second ${ }^{14}$ and the third ${ }^{15}$ generation of computational phantoms.

Both computational and physical phantoms are progressing, since their response can only be validated with one another. When it comes to radiation protection of the patients, new phantoms are not only providing data for new and improved internal dosimetry software, but also for dose optimization studies, dose planning, and research on the biological effects of ionizing radiation. ${ }^{12}$

\section{Internal dosimetry codes}

MIR dose: MIRDose was developed and distributed by the Radiation Internal Dose Information Center, Oak Ridge Institute for Science and Education, USA. The software contains tables of the $\mathrm{S}$ values for common radionuclides. The user must provide the biokinetic data in residence times for each source organs. It then generates tables of organ doses per unit administered activity in $\mathrm{rad} / \mathrm{mCi}$, and in International System (SI) units mGy/MBq. ${ }^{16,17}$

Versions 2 and 3 used pediatric mathematical phantom series for the photon-specific absorbed fraction libraries, giving the user the ability to calculate dose estimates for adults $(70 \mathrm{~kg}), 15$-yr-olds (57kg), 10-yr-olds (32kg), 5-yr-olds (19kg), 1-yr-olds $(9.2 \mathrm{~kg})$ and newborns $(3.4 \mathrm{~kg})$. These models were based on the 1974 adult phantom of Snyder et al. ${ }^{18}$ This adaptation is exemplified in Figure 2. The 15 age phantom was modified to represent both a 15 year-old male and an adult female. This was justified by the body weight and dimensions of a reference adult female from ICRP $1975,{ }^{12}$ which was close to those of the $15 \mathrm{yr}$-old. ${ }^{19,20}$ Figure 2 also shows the active bone marrow considered in the mathematical model from 1974 and the one considered in MIR dose software. ${ }^{20}$

Main features: The software has first-generation phantom libraries which allow the calculation of absorbed organ doses for individuals of different ages and sizes, and for women at different stages of pregnancy. ${ }^{1}$

Difficulties: The user must describe the biokinetics of the radiopharmaceutical $^{1}$ (from animal or human data) or find them in the literature. However, radiopharmaceuticals' kinetic models are constantly being updated as new data becomes available to improve them. Also, it is still questionable whether the model for the $15 \mathrm{yr}$-old should be used as an adult female. 

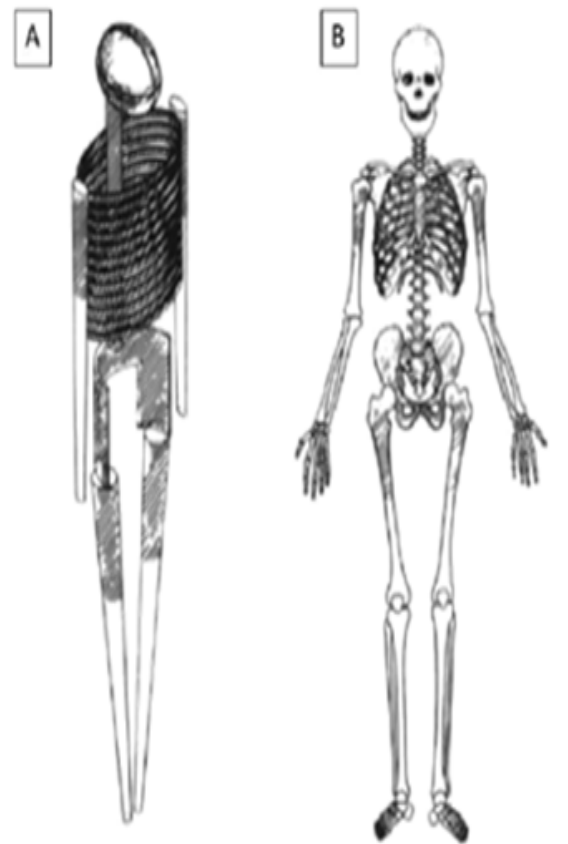

Figure 2 Mathematical phantoms from Snyder in 1974 - (A) and the one improved for MIRDose calculations (B). The shaded areas are the active bone marrow. $^{20}$

Currently, MIR Dose 3.0 and 3.1 codes are still being used for absorbed organ dose calculations. For instance, it is used to address a therapeutic dose in an organ after a tracer patient-specific dosimetry. ${ }^{21}$ Also for absorbed dose determination in non-target organs ${ }^{22}$ and for development of new radiopharmaceuticals. ${ }^{23}$

\section{MABDOSE}

MABDOSE was developed at the University of Colorado. It is another package based on fixed geometries.

Main features: It allows the user to place spherically shaped tumors within the simplified anatomic model originally described by MIRD Committee. Monte Carlo (MC) simulation was incorporated into this code. This method accommodates tumor dosimetry within the idealized geometry defined by the MIRD Committee. ${ }^{24,25}$ Unlike MIRDose 3 , it can estimate the dose to adjacent organs as a result of the tumor activity and the dose to the tumor as a result of activity in adjacent tissues by calculating $\mathrm{S}$ values for metastases as source or target organs, treating the tumor as a sphere within the Reference Man geometry and performing $\mathrm{MC}$ simulation in real time. ${ }^{26}$

Difficulties: It uses fixed geometries from the first-generation mathematical phantoms, hence it cannot be used for patient-specific dosimetry.

\section{OLINDA/EXM}

The code OLINDA/EXM, Organ Level INternal Dose Assessment/ EXponential Modeling, was designed as an update to MIR Dose.

Main features: The nuclear medicine dose factors were published by the same developers of MIRDose, but for many more nuclides (over 1000 vs 117), including some alpha emitters. ${ }^{27}$ It uses the second-generation voxel-based realistic phantoms for adults, children and pregnant women, based on ICRP 89 reference organ masses, besides animal models for mice, rats, and dogs. It can modify organ masses to patient-specific values and assign activity to the walls of hollow organs. ${ }^{28}$ In the EXM code portion, users can perform kinetic analyses, fitting sums of exponentials to data gathered in animal or human studies. ${ }^{28}$

Difficulties: Version 1 was FDA approved. However it was withdrawn from the market and replaced by Version 2, which is the only one currently available, and users also have to enter biokinetic data into the code.

\section{Tridimensional (3D) dosimetry codes}

3D patient-specific dosimetry better accounts for radionuclide distribution and anatomic patient variability. Cumulated activity data need to be input in these codes, which are usually obtained from SPECT and nuclear medicine planar imaging studies. ${ }^{29}$

\section{DOSIMG}

This software has been used with mathematical phantoms to analyze different quantitative SPECT algorithms on MC-derived absorbed dose calculations.

Main features: Mathematical phantoms are generated through CT and SPECT images and dose calculations derived from these are compared with "true" dose results derived from the actual mathematical phantom data. ${ }^{30}$ The methodology can be applied to $111 \mathrm{In} / 90 \mathrm{Y}$ dosimetry. $^{31}$

Difficulties: some inaccuracies were imposed by the SPECT system limited spatial resolution, for which the collimator response correction did not fully compensate. Compensation methods are necessary, based on physical models.

\section{Royal marsden dosimetry package-RMDP}

MIRD Committee has also published $\mathrm{S}$ value tabulations for different voxel sizes and source-target voxel distances. ${ }^{32}$ The resulting $S$ value tabulations reduce the lengthy and complex MC calculations to estimate absorbed dose. Since previously tabulated $\mathrm{S}$ values used a fixed anatomic model, geometries deviate significantly from one another. ${ }^{33}$ Voxel $\mathrm{S}$ values were developed to overcome this problem and have been adopted in several dose calculation programs, such as RMDP, a dedicated package for Iodine-131 (131I) therapy. ${ }^{34,35}$

Main features: it includes full photon interactions in a hexagonal-hole collimator and the gamma camera crystal. This code has led to improved 131I image quantification and contributes towards 3D dosimetry. ${ }^{36}$

Difficulties: Its accuracy is limited by the quality of the cumulated activity data that is entered in the code, which is the same difficulty found for most codes.

\section{Voxel dose}

This code was developed to calculate patient-specific 3D-dose maps at the voxel level. This software was validated with a physical abdominal phantom, the Liqui-Phil ${ }^{\mathrm{TM}}$, with four organ inserts and one spherical tumor filled with a known activity concentrations of Indium-111 (111In), combined with the Thermoluminescent Dosimetry technique (TLD) ${ }^{37}$

Main features: The SPECT acquisitions are reconstructed using a filtered back projection method, with attenuation and scatter correction; the 3D cumulated activity map is generated by integrating 
SPECT data; and a 3D dose map is computed by convolution using the Fourier Transform of the cumulated activity map and corresponding MIRD voxel $\mathrm{S}$ values. ${ }^{37}$

Difficulties: It could not precisely associate the position of the TLD to a voxel and it had limits of the quantification method, such as scatter correction and partial volume effect. ${ }^{37}$

\section{D-ID}

It is an ongoing-investigation code in $3 \mathrm{D}$ internal Dosimetry used for research. Multiple planar images are used combined with a single SPECT image. The planar images are used to obtain kinetic information, and SPECT images for better spatial distribution of radioactivity. ${ }^{29}$ This software introduced the concept of dose-volume histograms for internally administered radionuclides..$^{38}$ It may be used to perform both Monte Carlo and point-kernel-based calculations, and to examine the impact of different radionuclides on dose distribution, given a fixed cumulated activity distribution. ${ }^{29}$

Main features: it was able to perform a dosimetric analysis of 131I-labeled anti-CD20 antibody therapy for patients with nonHodgkin's lymphoma. It has also been showing that it is feasible to perform Iodine-124 (124I) PET-based patient-specific 3D dosimetry, and that sequential PET can be used to obtain cumulated activity images for 3D dosimetry. ${ }^{39}$

Difficulties: To obtain 3D cumulated activity images, the registered image sets need to be integrated, voxel by voxel, over time. Isodose contours need to be drawn over the images to provide information regarding absorbed dose distribution within the tumor volume. ${ }^{39}$

\section{RADAR}

It is a code that use anatomical models based on NURBS hybrid models to determine absorbed dose in tissues and organs. ${ }^{40}$

\section{JADA}

It is a MATLAB graphic interface that can be used to process data obtained by planar imaging or SPECT. Activity data in each time for source regions can be obtained by a set of tools. This software uses the same OLINDA phantoms and absorbed doses are also estimated by MC simulations. ${ }^{41}$

\section{CELLDOSE}

It is a C Language Code used to estimate absorbed dose from lowenergy-electron irradiation (from $10 \mathrm{eV}$ to $1 \mathrm{MeV}$ ) on a cell level. ${ }^{42}$

\section{DoRadlo}

The Ionizing Radiation Dosimetry (DoRadIo) is a code that receives information from source organs and target organs and generates graphical and numerical results. It was developed by MASH voxel simulation combined with EGSnrc MC code. Radiopharmaceutical biodistribution and kinetics are estimated through imaging and pixel intensity variation in a region of interest. ${ }^{43}$

\section{Occupational internal dosimetry codes}

Some codes have been created to estimate occupational radionuclides ingestion or inhalation, based on the International Commission on Radiological Protection (ICRP) published dose coefficients.

\section{Activity and internal dose estimates-AIDE}

This code is used for both in vivo biodistributions and in vitro assay data studies. Its main application is in radiation safety and estimates of absorbed doses associated with occupational ingestion of radionuclides. ${ }^{44}$

\section{Program for linear internal age-dependent doses- -PLEIADES}

Developed by the UK's Health Protection Agency, aims to solve the problems related to biokinetic. ${ }^{45}$

\section{Conclusion}

It is well-known that MIRD provides an average equivalent dose in the target organ and do not address the maximum absorbed dose. Spatial dose distribution can be achieved by voxels model simulation discriminating absorbed dose in a specific part of the organ such as an artery, for example. Such improvement can contribute to better specify deterministic effects of radiation in the organs. As nuclear medicine expands to include new therapeutic and diagnostic procedures, as well as new radiopharmaceuticals, the importance of radionuclide dosimetry has also been increasingly important. Radionuclide dosimetry should be used to plan radionuclide therapy in the same way that dosimetry is used to plan external beam radiotherapy. It is important to understand and distinguish the response between biological and dosimetry factors, to achieve treatment planning in nuclear medicine therapies. Therefore, it is essential to nuclear medicine community that ongoing improvements in dosimetry methodologies and codes continue so that the role of dosimetry, relative to biological factors could be properly evaluated.

\section{Acknowledgements}

We would like to thank the CAPES and CNPq programs for granting scholarships that allow us to continually work for the progress of science and knowledge for our country.

\section{Conflict of interest}

Author declares that there is no conflict of interest.

\section{References}

1. Stabin MG. MIRDOSE: Personel computer software for internal dose assessment in nuclear medicine. J Nucl Med. 1996;37(3):538-546.

2. Shahbazi-Gahrouei D, Nikzad S. Determination of organ doses in radioiodine therapy using medical internal radiation dosimetry (MIRD) method. Iranian J Radiat Res. 2011;8(1):249-252.

3. Weber D, Eckerman KF, Dillman LT, et al. MIRD: Radionuclide data and decay schemes. Society of Nuclear Medicine. New York; 1989

4. ICRP. Radionuclide transformations-energy and intensity of emissions. USA: Pergamon Press, Oxford; 1983.

5. Therapeutic applications of radiopharmaceuticals. India: Proceedings of an International Seminar held in Hyderabad. IAEA. 2001:18-22.

6. Silva CCO. Dosimetria pré-clínica no desenvolvimento de novos radiofármacos através de simulação por código de Monte Carlo. Master's Degree Dissertation From COPPE/UFRJ. 2017:33-34.

7. McParland BJ. Nuclear Medicine Radiation Dosimetry: Advanced Theoretical Principles. New York: Springer; 2010. 
8. Berdeguez MBT, Thomas S, Rafful P, et al. A new approach for radiosynoviorthesis: A dose-optimized planning method based on Monte Carlo simulation and synovial measurement using 3D slicer and MRI. Med Phys. 2017;44(7):3821-3829.

9. Snyder WS, Ford MR, Warner GG, et al. MIRD5: Estimates of Absorbed Fractions for Mono-energetic Photon Sources Uniformly Distributed in Various Organs of a Heterogeneous Phantom. J Nucl Med. 1969;10(3):5-52.

10. Caon M. Voxel-based computational models of real human anatomy: a review Rad. Radiat Environ Biophys. 2004;42(4):229-235.

11. Lee C, Wesley DL E. Bolch NURBS-based 3-d anthropomorphic computational phantoms for radiation dosimetry applications. Radiat Prot Dosimetry. 2007;127(1-4):227-232.

12. Ramos SMO, Thomas S, Berdeguez MBT, et al. Anthropomorphic Phantoms - Potential for More Studies and Training in Radiology. Int $J$ Radiol Radiat Ther. 2017;2(4):00033.

13. Kramer R. The calculation of dose from external photon exposures using reference human phantoms and Monte Carlo methods: Part I. The male (ADAM) and female (EVA) adult mathematical phantoms, GSFReport S-885, Neuherberg-Muenchen: Institut fuer Strahlenschutz, GSFForschungszentrum fuer Umwelt und Gesundheit. 1982.

14. Kramer R. All about FAX: a Female Adult voXel phantom for Monte Carlo calculation in radiation protection dosimetry. Phys Med Biol. 2004;49(23):5203-5216.

15. Hoseinian-Azghadi E, Rafat-Motavalli L, Miri-Hakimabad H. Development of a 9-months pregnant hybrid phantom and its internal dosimetry for thyroid agents. J Radiat Res. 2014;55(4):730-747.

16. Stabin MG, Flux GD. Internal dosimetry as a tool for radiation protection of the patient in nuclear medicine. BIJJ. 2007;3:e28.

17. Fleming JS. A technique for the absolute measurement of activity using a gamma camera and computer. Phys Med Biol. 1979;24(1):176-180.

18. Snyder WS, Ford MR, Warner GG, et al. A tabulation of dose equivalent per microcurie-day for source and target organs of an adult for various radionuclides: Part 1. Oak Ridge National Laboratory Rep, ORNL-5000. 1974.

19. ICRP. Report of the task group on Reference Man. International Commission on Radiological Protection. Oxford, USA: Pergamon Press; 1975.

20. Cristy M, Eckerman K. Specific absorbed fractions of energy at various ages from internal photons sources. Oak Ridge National Laboratory, USA; 1987.

21. Changmuang W, Kositwattanarerk A, Thongklam A, et al. Radiation dosimetry in non-hodgkin's b-cell lymphoma patients treated with 131i-rituximab radioimmunotherapy. Ramathibodi Medical Journal. 2017;40(2):2.

22. Shanei A, Heydari F, Moslehi M. Determination of Uterus Absorbed Dose by Patients following Myocardial Perfusion Scan using TLD and Conjugate View Methods. J Biomed Phys Eng. 2016.

23. Deilami-nezhada L, Moghaddam-Banaemb L, Sadeghic M. Development of bone seeker radiopharmaceuticals by Scandium-47 and estimation of human absorbed dose. Appl Radiat Isot. 2017;129:108-116.

24. Johnson TK, McClure D, McCourt S. MABDOSE. I: characterization of a general purpose dose estimation code. Med Phys. 1999;26(7):1389-1395.

25. Johnson TK, McClure D, McCourt S. MABDOSE. II: validation of a general purpose dose estimation code. Med Phys. 1999;26(7):1396-1403.

26. Clairand I, Marcel R, Gouriou J, et al. DOSE3D: EGS4 Monte Carlo code-based software for internal radionuclide dosimetry. J Nucl Med. 1999;40(9):1517-1523.
27. Stabin MG, Siegel JA. Physical models and dose factors for use in internal dose assessment. Health Phys. 2003;85(3):294-310.

28. http://www.doseinfo-radar.com/OLINDA.html

29. Sgouros G, Kolbert KS. The three-dimensional internal dosimetry software package, 3D-ID. In: Zaidi H, Sgouros G, editors. Therapeutic Applications of Monte Carlo Calculations in Nuclear Medicine. Philadelphia, USA; 2002. p. 249-258.

30. Ljungberg M, Sjogreen K, Liu X, et al. A 3-dimensional absorbed dose calculation method based on quantitative SPECT for radionuclide therapy: evaluation for 131I using Monte Carlo simulation. $J$ Nucl Med. 2002;43(8):1101-1109.

31. Ljungberg M, Frey E, Sjogreen K, et al. 3D absorbed dose calculations based on SPECT: evaluation for 111-In/90-Y therapy using Monte Carlo simulations. Cancer Biother Radiopharm. 2003;18(1):99-107.

32. Bolch WE, Bouchet LG, Robertson JS, et al. MIRD pamphlet no. 17: the dosimetry of nonuniform activity distributions-radionuclide $\mathrm{S}$ values at the voxel level. J Nucl Med. 1999;40(1):11S-36S.

33. McKay E. A software tool for specifying voxel models for dosimetry estimation. Cancer Biother Radiopharm. 2003;18(3):379-392.

34. Guy MJ, Flux GD, Papavasileiou P, et al. RMDP: a dedicated package for 131I SPECT quantification, registration and patient-specific dosimetry. Cancer Biother Radiopharm. 2003;18(1):61-69.

35. Williams LE, Liu A, Raubitschek AA, et al. A method for patient-specific absorbed dose estimation for internal beta emitters. Clin Cancer Res. 1999;5(10):3015s-3019s

36. Guy MJ, Flux GD, Papavasileiou P, et al. RMDP: a dedicated package for 131I SPECT quantification, registration and patient-specific dosimetry. Cancer Biother Radiopharm. 2003;18(1):61-69.

37. Gardin I, Bouchet LG, Assié K, et al. Cancer Biotherapy and Radiopharmaceuticals. Libert. 2004;18(1):109-115.

38. Kolbert KS, Sgouros G, Scott AM. Dose-volume histogram representation of patient dose distribution in 3-dimensional internal dosimetry [abstract]. J Nucl Med. 1994;35:123-124.

39. Kolbert KS, Sheikh A, Pentlow KS, et al. Patient-Specific Dosimetry for 131I Thyroid Cancer Therapy Using 124I PET and 3-DimensionalInternal Dosimetry (3D-ID) Software. George Sgouros, PhD1, 5MD2. J Nucl Med. 2004;45(8):1366-1372.

40. Stabin MG, Xu GX, Emmons MA, et al. RADAR Reference Adult, Pediatric, and Pregnant Female Phantom Series for Internal and External Dosimetry. Journal of Nuclear Medicine. 2012;53(11):1807-1813.

41. Grimes J, Uribe C, Celler A. JADA: A graphical interface for comprehensive internal dose assessment in nuclear medicine. Med Phys. 2013;40(7):072501.

42. Champion C, Zanotti-Gregonara P, Hindié E. CELLDOSE: A Monte Carlo Code to Assess Electron Dose Distribution - S values for 131I in Spheres of Varios Sizes. Journal of Nuclear Medicine. 2008;49:151-157.

43. Leal NETO V, Vieira JW, Lima FRA. Aplicativo para dosimetria interna usando a distribuição biocinética de fótons baseada em imagens de Medicina Nucelar. Radiologia Brasileira. 2014;47(5):275-282.

44. Bertelli L, Melo DR, Lipsztein J, et al. AIDE: internal dosimetry software. Radiat Prot Dosimetry. 2008;130(3):358-367.

45. Fell TP, Phipps AW, Smith TJ. The internal dosimetry code PLEIADES Radiat Prot Dosimetry. 2007;124(4):327-338. 